Bull. Korean Math. Soc. 48 (2011), No. 6, pp. 1271-1290

http://dx.doi.org/10.4134/BKMS.2011.48.6.1271

\title{
CONTROLLABILITY OF SECOND-ORDER IMPULSIVE FUNCTIONAL DIFFERENTIAL EQUATIONS WITH STATE-DEPENDENT DELAY
}

\author{
Ganesan Arthi and Krishnan Balachandran
}

\begin{abstract}
The purpose of this paper is to investigate the controllability of certain types of second order nonlinear impulsive systems with statedependent delay. Sufficient conditions are formulated and the results are established by using a fixed point approach and the cosine function theory. Finally examples are presented to illustrate the theory.
\end{abstract}

\section{Introduction}

In this paper, we establish sufficient conditions for the controllability of nonlinear second order impulsive functional differential equations with statedependent delay. More precisely, we consider the following abstract control system:

$$
\begin{aligned}
x^{\prime \prime}(t) & =A x(t)+B u(t)+f\left(t, x_{\rho\left(t, x_{t}\right)}\right), t \in I=[0, a], t \neq t_{i}, \\
x_{0} & =\varphi \in \mathcal{B}, \quad x^{\prime}(0)=\eta \in X, \\
\triangle x\left(t_{i}\right) & =I_{i}\left(x_{t_{i}}\right), i=1,2, \ldots, n, \\
\triangle x^{\prime}\left(t_{i}\right) & =J_{i}\left(x_{t_{i}}\right), i=1,2, \ldots, n,
\end{aligned}
$$

where $A$ is the infinitesimal generator of a strongly continuous cosine family of bounded linear operators $(C(t))_{t \in \mathbb{R}}$ defined on a Banach space $X$. The control function $u(\cdot)$ is given in $L^{2}(I, U)$, a Banach space of admissible control functions with $U$ as a Banach space and $B: U \rightarrow X$ as a bounded linear operator; the function $x_{t}:(-\infty, 0] \rightarrow X, x_{t}(\theta)=x(t+\theta)$, belongs to some abstract phase space $\mathcal{B}$ described axiomatically; $0<t_{1}<\cdots<t_{n}<a$ are prefixed numbers; $f: I \times \mathcal{B} \rightarrow X, \rho: I \times \mathcal{B} \rightarrow(-\infty, a], I_{i}(\cdot): \mathcal{B} \rightarrow X, J_{i}(\cdot): \mathcal{B} \rightarrow X$ are appropriate functions and the symbol $\triangle \xi(t)$ represents the jump of the function $\xi(\cdot)$ at $t$, which is defined by $\triangle \xi(t)=\xi\left(t^{+}\right)-\xi\left(t^{-}\right)$.

Received July 22, 2010.

2010 Mathematics Subject Classification. 93B05, 34A37.

Key words and phrases. controllability, impulsive differential equations, neutral differential equations, state-dependent delay. 
Impulsive differential equations arise naturally from a wide variety of applications such as aircraft control, inspection process in operation research, population dynamics, drug administration and threshold theory in biology. That is why in recent years they form an object of investigations. However, the control theory of impulsive differential equations has not yet been sufficiently studied elaborately compared to that of ordinary differential equations. The concept of controllability plays an important role in the analysis and design of control systems. Controllability of nonlinear systems with and without impulses has been studied by many authors [3, 22, 26-28, 30]. For more details on impulsive differential equations and on their applications, we refer to the monographs of Lakshmikantham et al. [24] and Samoilenko and Perestyuk [31] and the references therein. Neutral differential systems with impulses arise in many areas of applied mathematics and these systems have been extensively investigated during the last decades.

On the other hand, delay-differential equations form one of the oldest branches of the theory of infinite-dimensional dynamical systems - theory which describes qualitative properties of systems, changing in time. Recently a new class of delay equations - equations with state-dependent delay (SDD) has attracted much attention of researchers. Functional differential equations with statedependent delay have become more important in some mathematical models of real phenomena. The reader is referred to $[1,4,5,7,8,11-14,32]$ and references therein for some examples and applications. The problem of the existence of solutions of functional differential equations with state-dependent delay has been treated recently in $[2,16-20,25]$. The literature related to second order nonlinear systems with state-dependent delay is not vast, to our knowledge, in the recent works $[6,15]$. We also cite $[9,12,35]$ for the case of neutral differential equations with dependent delay. To the best of our knowledge, the study of controllability of abstract nonlinear second order impulsive systems with state-dependent delay is an almost untreated topic in the literature and this fact is the main motivation for this article.

The rest of this article is organized as follows. In Section 2, we introduce some notations and necessary preliminaries. In Section 3, we establish the controllability results for the abstract Cauchy problem. In Section 4, we present some examples to show the application of the results.

\section{Preliminaries}

In what follows we recall some definitions, notations, lemmas and results that we need in the sequel.

Throughout this paper, $(X,\|\cdot\|)$ is a Banach space and $A$ is the infinitesimal generator of a strongly continuous cosine family of bounded linear operators $(C(t))_{t \in \mathbb{R}}$ on Banach space $X$. We denote by $(S(t))_{t \in \mathbb{R}}$ the sine function associated with $(C(t))_{t \in \mathbb{R}}$ which is defined by $S(t) x=\int_{0}^{t} C(s) x d s$ for $x \in X$ and $t \in \mathbb{R}$. 
The notation $[D(A)]$ stands for the domain of the operator $A$ endowed with the graph norm $\|x\|_{A}=\|x\|+\|A x\|, x \in D(A)$. Moreover, in this work, $E$ is the space formed by the vectors $x \in X$ for which $C(\cdot) x$ is of class $C^{1}$ on $\mathbb{R}$. It was proved by Kisynski [23] that $E$ endowed with the norm $\|x\|_{E}=$ $\|x\|+\sup _{0 \leq t \leq 1}\|A S(t) x\|, x \in E$, is a Banach space. The operator valued function

$$
\mathcal{H}(t)=\left[\begin{array}{ll}
C(t) & S(t) \\
A S(t) & C(t)
\end{array}\right]
$$

is a strongly continuous group of bounded linear operators on the space $E \times X$ generated by the operator $\mathcal{A}=\left[\begin{array}{cc}0 & I \\ A & 0\end{array}\right]$ defined on $D(A) \times E$. From this, it follows that $A S(t): E \rightarrow X$ is a bounded linear operator and that $A S(t) x \rightarrow 0, t \rightarrow 0$, for each $x \in E$. Furthermore, if $x:[0, \infty) \rightarrow X$ is a locally integrable function, then the function $y(t)=\int_{0}^{t} S(t-s) x(s) d s$ defines an $E$-valued continuous function. This assertion is a consequence of the fact that

$$
\int_{0}^{t} \mathcal{H}(t-s)\left[\begin{array}{l}
0 \\
x(s)
\end{array}\right] d s=\left[\int_{0}^{t} S(t-s) x(s) d s, \int_{0}^{t} C(t-s) x(s) d s\right]^{T}
$$

defines an $E \times X$-valued continuous function.

The existence of solutions for the second order abstract Cauchy problem

$$
\begin{aligned}
x^{\prime \prime}(t) & =A x(t)+g(t), \quad 0 \leq t \leq a, \\
x(0) & =u, \quad x^{\prime}(0)=v,
\end{aligned}
$$

where $g: I \rightarrow X$ is an integrable function, has been discussed in [33]. Similarly the existence of solutions of semilinear second order abstract Cauchy problems has been treated in [34]. We only mention here that the function $x(\cdot)$ given by

$$
x(t)=C(t) u+S(t) v+\int_{0}^{t} S(t-s) g(s) d s, \quad 0 \leq t \leq a,
$$

is called a mild solution of (5)-(6) and that when $u \in E, x(\cdot)$ is continuously differentiable and

$$
x^{\prime}(t)=A S(t) u+C(t) v+\int_{0}^{t} C(t-s) g(s) d s, \quad 0 \leq t \leq a .
$$

For additional details on the cosine function theory, we refer the reader to [10, 33, 34].

To consider the impulsive conditions (3)-(4), it is convenient to introduce some additional concepts and notations.

A function $u:[\mu, \tau] \rightarrow X$ is said to be a normalized piecewise continuous function on $[\mu, \tau]$ if $u$ is piecewise continuous and left continuous on $(\mu, \tau]$. We denote by $\mathcal{P C}([\mu, \tau], X)$ the space of normalized piecewise continuous functions from $[\mu, \tau]$ into $X$. In particular, we introduce the space $\mathcal{P C}$ formed by all normalized piecewise continuous functions $u:[0, a] \rightarrow X$ such that $u(\cdot)$ is continuous at $t \neq t_{i}, u\left(t_{i}^{-}\right)=u\left(t_{i}\right)$ and $u\left(t_{i}^{+}\right)$exists, for $i=1,2, \ldots, n$. In this paper, we always assume that $\mathcal{P C}$ is endowed with the norm $\|u\|_{\mathcal{P C}}=$ $\sup _{s \in I}\|u(s)\|$. It is clear that $\left(\mathcal{P C},\|\cdot\|_{\mathcal{P C}}\right)$ is a Banach space. 
In what follows, we put $t_{0}=0, t_{n+1}=a$ and, for $u \in \mathcal{P C}$, we denote by $\tilde{u}_{i}$, for $i=0,1, \ldots, n-1$, the function $\tilde{u}_{i} \in C\left(\left[t_{i}, t_{i+1}\right] ; X\right)$ given by $\tilde{u}_{i}(t)=u(t)$ for $t \in\left(t_{i}, t_{i+1}\right]$ and $\tilde{u}_{i}\left(t_{i}\right)=\lim _{t \rightarrow t_{i}^{+}} u(t)$. Moreover, for a set $B \subseteq \mathcal{P C}$, we denote by $\widetilde{B}_{i}$, for $i=0,1, \ldots, n-1$, the set $\widetilde{B}_{i}=\left\{\widetilde{u}_{i}: u \in B\right\}$. We will herein define the phase space $\mathcal{B}$ axiomatically, using ideas and notations developed in [21] and suitably modify to treat retarded impulsive differential equations. More precisely, $\mathcal{B}$ will denote the vector space of functions defined from $(-\infty, 0]$ into $X$ endowed with a seminorm denoted $\|\cdot\|_{\mathcal{B}}$ and such that the following axioms hold:

(A) If $x:(-\infty, \mu+b] \rightarrow X, b>0$, is a function such that $x_{\mu} \in \mathcal{B}$ and $\left.x\right|_{[\mu, \mu+b]} \in \mathcal{P C}([\mu, \mu+b], X)$, then, for every $t \in[\mu, \mu+b)$, the following conditions hold:

(i) $x_{t}$ is in $\mathcal{B}$,

(ii) $\|x(t)\| \leq H\left\|x_{t}\right\|_{\mathcal{B}}$,

(iii) $\left\|x_{t}\right\|_{\mathcal{B}} \leq K(t-\mu)$ sup $\{\|x(s)\|: \mu \leq s \leq t\}+M(t-\mu)\left\|x_{\mu}\right\|_{\mathcal{B}}$,

where $H>0$ is a constant; $K, M:[0, \infty) \rightarrow[1, \infty), K$ is continuous, $M$ is locally bounded and $H, K, M$ are independent of $x(\cdot)$.

(B) The space $\mathcal{B}$ is complete.

Now we consider some examples of phase spaces.

Example 2.1. The phase space $\mathcal{P C}(X)$.

A function $\psi:(-\infty, 0] \rightarrow X$ is said to be normalized piecewise continuous if $\psi$ is left continuous and the restriction of $\psi$ to any interval $[-r, 0]$ is piecewise continuous. Let $h:(-\infty, 0] \rightarrow[1, \infty)$ be a continuous nondecreasing function which satisfies the conditions (g-1), (g-2) in the terminology of [21]. Next we slightly modify the definition of spaces $c_{h}, c_{h}^{0}$ in [21]. We denote, by $\mathcal{P C}_{h}(X)$, the space formed by the normalized piecewise continuous functions $\psi$ such that $\psi / h$ is bounded on $(-\infty, 0]$ and, by $\mathcal{P} \mathcal{C}_{h}^{0}(X)$, the subspace of $\mathcal{P} \mathcal{C}_{h}(X)$ consisting of functions $\psi$ such that $[\psi(\theta) / h(\theta)] \rightarrow 0$ as $\theta \rightarrow-\infty$. It is easy to see that $\mathcal{B}=$ $\mathcal{P C}_{h}(X)$ and $\mathcal{B}=\mathcal{P} \mathcal{C}_{h}^{0}(X)$ endowed with the norm $\|\psi\|_{\mathcal{B}}:=\sup _{\theta \in(-\infty, 0]} \frac{\|\psi(\theta)\|}{h(\theta)}$ are phase spaces in the sense defined above.

Example 2.2. The phase space $\mathcal{P C} r \times L^{p}(h, X)$.

Let $r \geq 0,1 \leq p<\infty$ and let $h(\cdot):(-\infty,-r] \rightarrow \mathbb{R}$ be a non-negative measurable function which satisfies the conditions (g-5), (g-6) in the terminology of [21]. Briefly this means that $h$ is locally integrable and there exists a non-negative, locally bounded function $\gamma(\cdot)$ on $(-\infty, 0]$ such that $h(\xi+\theta) \leq$ $\gamma(\xi) h(\theta)$ for all $\xi \leq 0$ and $\theta \in(-\infty,-r) \backslash N_{\xi}$, where $N_{\xi} \subseteq(-\infty,-r)$ is a set with Lebesgue measure zero. The space $\mathcal{B}=\mathcal{P} \mathcal{C}_{r} \times L^{p}(h, X)$ consists of all classes of Lebesgue-measurable functions $\varphi:(-\infty, 0] \rightarrow X$ such that $\left.\varphi\right|_{[-r, 0]} \in \mathcal{P C}([-r, 0], X)$ and $h\|\varphi\|^{p}$ is Lebesgue integrable on $(-\infty,-r)$. The seminorm in this space is defined by

$$
\|\varphi\|_{\mathcal{B}}=\sup \{\|\varphi(\theta)\|:-r \leq \theta \leq 0\}+\left(\int_{-\infty}^{-r} h(\theta)\|\varphi(\theta)\|^{p} d \theta\right)^{1 / p} .
$$


Proceeding, as in the proof of [21, Theorem 1.3.8], it follows that $\mathcal{B}$ is a space which verifies the axioms (A) and (B). Moreover, when $r=0$, this space coincides with $C_{0} \times L^{p}(h, X)$ and if, in addition $p=2$, we can take $H=$ $1, K(t)=\left(1+\int_{-t}^{0} h(\theta) d \theta\right)^{1 / 2}$ and $M(t)=\gamma(-t)^{1 / 2}$ for $t \geq 0$.

Remark 2.3. Let $\psi \in \mathcal{B}$ and $t \leq 0$. The notation $\psi_{t}$ represents the function defined by $\psi_{t}(\theta)=\psi(t+\theta)$. Consequently if the function $x(\cdot)$ in the axiom $(A)$ is such that $x_{0}=\psi$, then $x_{t}=\psi_{t}$. We observe that $\psi_{t}$ is well defined for $t<0$, since the domain of $\psi$ is $(-\infty, 0]$. We also note that, in general, $\psi_{t} \notin \mathcal{B}$; consider, for example, functions of the type $x^{\mu}(t)=(t-\mu)^{-\alpha} \chi_{(\mu, 0]}, \mu>0$, where $\chi_{(\mu, 0]}$ is the characteristic function of $(\mu, 0], \mu<-r$ and $\alpha p \in(0,1)$, in the space $\mathcal{P} \mathcal{C}_{r} \times L^{p}(h ; X)$.

The terminology and notations are those generally used in functional analysis. In particular, for Banach spaces $\left(Z,\|\cdot\|_{Z}\right),\left(W,\|\cdot\|_{W}\right)$, the notation $\mathcal{L}(Z, W)$ stands for the Banach space of bounded linear operators from $Z$ into $W$ and we abbreviate to $\mathcal{L}(Z)$ whenever $Z=W$. Moreover $B_{r}(x: Z)$ denotes the closed ball with center at $x$ and radius $r>0$ in $Z$. Additionally, for a bounded function $\xi: I \rightarrow Z$ and $0 \leq t \leq a$, we employ the notation $\|\xi\|_{t}$ for

$$
\|\xi\|_{t}=\sup \{\|\xi(s)\|: s \in[0, t]\} .
$$

In particular, if $M(\cdot), K(\cdot)$ are the functions in the axiom $(A)$, then $M_{a}=$ $\sup _{t \in I} M(t)$ and $K_{a}=\sup _{t \in I} K(t)$.

For completeness, we include the following well-known results.

Lemma 2.4 ([26, Lemma 3.1.]). Assume that (Hf1), $(H f 2),(W)$ hold. Then the operator

$$
N y(t)=\int_{0}^{t} S(t-s)\left[f(s, y(s))+\left(\mathcal{B} u_{y}\right)(s)\right] d s, \quad t \in[0, b],
$$

is completely continuous.

Lemma 2.5 ([29, Sadovskii's Fixed Point Theorem]). Let $F$ be a condensing operator on a Banach space $X$. If $F(S) \subset S$ for a convex, closed and bounded set $S$ of $X$, then $F$ has a fixed point in $S$.

\section{Controllability results}

In this section, we study the controllability results for the abstract nonlinear second order impulsive systems. Along this section $N$ and $\tilde{N}$ are positive constants such that $\|C(t)\| \leq N$ and $\|S(t)\| \leq \tilde{N}$ for every $t \in I$. To prove our results, we always assume that $\rho: I \times \mathcal{B} \rightarrow(-\infty, a]$ is continuous and that $\varphi \in \mathcal{B}$.

In the sequel, we introduce the following conditions:

$\left(\mathbf{H}_{\varphi}\right)$ Let $\mathcal{R}\left(\rho^{-}\right)=\{\rho(s, \psi):(s, \psi) \in I \times \mathcal{B}, \rho(s, \psi) \leq 0\}$. The function $t \rightarrow$ $\varphi_{t}$ is well defined from $\mathcal{R}\left(\rho^{-}\right)$into $\mathcal{B}$ and there exists a continuous and 
bounded function $J^{\varphi}: \mathcal{R}\left(\rho^{-}\right) \rightarrow(0, \infty)$ such that $\left\|\varphi_{t}\right\|_{\mathcal{B}} \leq J^{\varphi}(t)\|\varphi\|_{\mathcal{B}}$ for every $t \in \mathcal{R}\left(\rho^{-}\right)$.

(H1) The function $f: I \times \mathcal{B} \rightarrow X$ satisfies the following conditions:

(i) Let $x:(-\infty, a] \rightarrow X$ be such that $x_{0}=\varphi$ and $\left.x\right|_{I} \in \mathcal{P C}$. The function $t \rightarrow f\left(t, x_{\rho\left(t, x_{t}\right)}\right)$ is measurable on $I$ and the function $t \rightarrow f\left(s, x_{t}\right)$ is continuous on $\mathcal{R}\left(\rho^{-}\right) \cup I$ for every $s \in I$.

(ii) For each $t \in I$, the function $f(t, \cdot): \mathcal{B} \rightarrow X$ is completely continuous.

(iii) There exist an integrable function $m: I \rightarrow[0, \infty)$ and a continuous non-decreasing function $W:[0, \infty) \rightarrow(0, \infty)$ such that, for every $(t, \psi) \in I \times \mathcal{B}$,

$$
\|f(t, \psi)\| \leq m(t) W\left(\|\psi\|_{\mathcal{B}}\right), \quad \liminf _{\xi \rightarrow \infty} \frac{W(\xi)}{\xi}=\Lambda<\infty .
$$

(iv) For every positive constant $r$, there exists an $\alpha_{r} \in L^{1}(I)$ such that

$$
\sup _{\|\psi\| \leq r}\|f(t, \psi)\| \leq \alpha_{r}(t) .
$$

(H2) $B$ is a continuous operator from $U$ to $X$ and the linear operator $W$ : $L^{2}(I, U) \rightarrow X$, defined by

$$
W u=\int_{0}^{a} S(a-s) B u(s) d s,
$$

has a bounded invertible operator $W^{-1}$ which takes values in

$$
L^{2}(I, U) / \operatorname{ker} W
$$

such that $\|B\| \leq M_{1}$ and $\left\|W^{-1}\right\| \leq M_{2}$ for some positive constants $M_{1}, M_{2}$.

(H3) There are positive constants $L_{I_{i}}, L_{J_{i}}$ such that

$$
\begin{aligned}
\left\|I_{i}\left(\psi_{1}\right)-I_{i}\left(\psi_{2}\right)\right\| \leq L_{I_{i}}\left\|\psi_{1}-\psi_{2}\right\|_{\mathcal{B}}, \quad \psi_{j} \in \mathcal{B}, \quad j=1,2, \quad i=1,2, \ldots, n, \\
\left\|J_{i}\left(\psi_{1}\right)-J_{i}\left(\psi_{2}\right)\right\| \leq L_{J_{i}}\left\|\psi_{1}-\psi_{2}\right\|_{\mathcal{B}}, \quad \psi_{j} \in \mathcal{B}, \quad j=1,2, \quad i=1,2, \ldots, n .
\end{aligned}
$$

(H4) The maps $I_{i}, J_{i}: \mathcal{B} \rightarrow X, i=1,2, \ldots, n$ are completely continuous and there exist continuous non-decreasing functions $\Phi_{i}, \Gamma_{i}:[0, \infty) \rightarrow$ $(0, \infty), i=1,2, \ldots, n$, such that

$$
\begin{aligned}
\left\|I_{i}(\psi)\right\| \leq \Phi_{i}\left(\|\psi\|_{\mathcal{B}}\right), & \liminf _{\zeta \rightarrow+\infty} \frac{\Phi_{i}(\zeta)}{\zeta}=\zeta_{i}<\infty, \\
\left\|J_{i}(\psi)\right\| \leq \Gamma_{i}\left(\|\psi\|_{\mathcal{B}}\right), & \liminf _{\zeta \rightarrow+\infty} \frac{\Gamma_{i}(\zeta)}{\zeta}=\sigma_{i}<\infty .
\end{aligned}
$$

Remark 3.1. The condition $\left(\mathrm{H}_{\varphi}\right)$ is frequently satisfied by functions that are continuous and bounded. In fact, assume that the space of continuous and 
bounded functions $C_{b}((-\infty, 0], X)$ is continuously included in $\mathcal{B}$. Then there exists $L>0$ such that

$$
\left\|\varphi_{t}\right\|_{\mathcal{B}} \leq L \frac{\sup _{\theta \leq 0}\|\varphi(\theta)\|}{\|\varphi\|_{\mathcal{B}}}\|\varphi\|_{\mathcal{B}}, \quad t \leq 0, \varphi \neq 0, \varphi \in C_{b}((-\infty, 0], X) .
$$

It is easy to see that the space $C_{b}((-\infty, 0], X)$ is continuously included in $\mathcal{P C}_{h}(X)$ and $\mathcal{P C}_{h}^{0}(X)$. Moreover, if $h(\cdot)$ verifies $(\mathrm{g}-5),(\mathrm{g}-6)$ in [21] and $h(\cdot)$ is integrable on $(-\infty,-r]$, then the space $C_{b}((-\infty, 0], X)$ is also continuously included in $\mathcal{P C}_{r} \times L^{p}(h ; X)$. For complementary details related to this matter, see Proposition 7.1.1 and Theorems 1.3.2 and 1.3.8 in [21].

Remark 3.2. In the rest of this paper, $y:(-\infty, a] \rightarrow X$ is the function defined by $y(t)=\varphi(t)$ on $(-\infty, 0]$ and $y(t)=C(t) \varphi(0)+S(t) \eta$ for $t \in I$. Also $\|y\|_{a}, M_{a}, K_{a}$ and $J_{0}^{\varphi}$ are the constants defined by $\|y\|_{a}=\sup _{s \in[0, a]}\|y(s)\|$, $M_{a}=\sup _{s \in[0, a]} M(s), K_{a}=\sup _{s \in[0, a]} K(s), J_{0}^{\varphi}=\sup _{t \in \mathcal{R}\left(\rho^{-}\right)} J^{\varphi}(t)$.

Definition 3.3. The system (1)-(4) is said to be controllable on the interval $I$, if for every $\varphi \in D(A), \eta \in E$ and $x_{1} \in X$, there exists a control $u \in L^{2}(I, U)$ such that the mild solution $x(t)$ of (1)-(4) satisfies $x(a)=x_{1}$.

Lemma 3.4 ([19, Lemma 2.1]). Let $x:(-\infty, a] \rightarrow X$ be a function such that $x_{0}=\varphi$ and $\left.x(\cdot)\right|_{I} \in \mathcal{P C}$. Then

$\left\|x_{s}\right\|_{\mathcal{B}} \leq\left(M_{a}+J_{0}^{\varphi}\right)\|\varphi\|_{\mathcal{B}}+K_{a} \sup \{\|x(\theta)\| ; \theta \in[0, \max \{0, s\}]\}, s \in \mathcal{R}\left(\rho^{-}\right) \cup I$.

Now we prove our main results.

\subsection{Second-order impulsive systems}

In this section, we study an impulsive control problem with state-dependent delay of the form (1)-(4). Motivated by (7), we introduce the following concept of mild solutions for the system (1)-(4).

Definition 3.5. A function $x:(-\infty, a] \rightarrow X$ is called a mild solution of the impulsive abstract Cauchy problem (1)-(4), if $x_{0}=\varphi, x_{\rho\left(s, x_{s}\right)} \in \mathcal{B}$ for every $s \in I ;\left.x(\cdot)\right|_{I} \in \mathcal{P C}$ and

$$
\begin{aligned}
x(t)= & C(t) \varphi(0)+S(t) \eta+\int_{0}^{t} S(t-s)\left[B u(s)+f\left(s, x_{\rho\left(s, x_{s}\right)}\right)\right] d s \\
& +\sum_{0<t_{i}<t} C\left(t-t_{i}\right) I_{i}\left(x_{t_{i}}\right)+\sum_{0<t_{i}<t} S\left(t-t_{i}\right) J_{i}\left(x_{t_{i}}\right), \quad t \in I .
\end{aligned}
$$

Theorem 3.6. Let the conditions $\left(\mathrm{H}_{\varphi}\right)$, ( $\left.\mathrm{H} 1\right)-(\mathrm{H} 4)$ hold. Then the system (1)-(4) is controllable on $(-\infty, a]$ provided that

$$
\left(1+a \tilde{N} M_{1} M_{2}\right)\left[K_{a}\left(\tilde{N} \Lambda \int_{0}^{a} m(s) d s+\sum_{i=1}^{n}\left(N L_{I_{i}}+\tilde{N} L_{J_{i}}\right)\right)\right]<1 .
$$


Proof. Consider the space $Y=\{x \in \mathcal{P C}: u(0)=\varphi(0)\}$ endowed with the uniform convergence topology. Using the assumption (H2), for an arbitrary function $x(\cdot)$, we define the control

$$
\begin{aligned}
u(t)= & W^{-1}\left[x_{1}-C(a) \varphi(0)-S(a) \eta-\int_{0}^{a} S(a-s) f\left(s, x_{\rho\left(s, x_{s}\right)}\right) d s\right. \\
& \left.-\sum_{i=1}^{n} C\left(a-t_{i}\right) I_{i}\left(x_{t_{i}}\right)-\sum_{i=1}^{n} S\left(a-t_{i}\right) J_{i}\left(x_{t_{i}}\right)\right](t) .
\end{aligned}
$$

Using this control, we shall show that the operator $\Psi: Y \rightarrow Y$ defined by

$$
\begin{aligned}
\Psi x(t)= & C(t) \varphi(0)+S(t) \eta+\int_{0}^{t} S(t-s) f\left(s, \bar{x}_{\rho\left(s, \bar{x}_{s}\right)}\right) d s \\
& +\int_{0}^{t} S(t-\xi) B W^{-1}\left[x_{1}-C(a) \varphi(0)-S(a) \eta\right. \\
& -\int_{0}^{a} S(a-s) f\left(s, \bar{x}_{\rho\left(s, \bar{x}_{s}\right)}\right) d s-\sum_{i=1}^{n} C\left(a-t_{i}\right) I_{i}\left(\bar{x}_{t_{i}}\right) \\
& \left.-\sum_{i=1}^{n} S\left(a-t_{i}\right) J_{i}\left(\bar{x}_{t_{i}}\right)\right](\xi) d \xi+\sum_{0<t_{i}<t} C\left(t-t_{i}\right) I_{i}\left(\bar{x}_{t_{i}}\right) \\
& +\sum_{0<t_{i}<t} S\left(t-t_{i}\right) J_{i}\left(\bar{x}_{t_{i}}\right), \quad t \in I,
\end{aligned}
$$

has a fixed point $x(\cdot)$. This fixed point $x(\cdot)$ is then a mild solution of the system (1)-(4). Clearly, $(\Psi x)(a)=x_{1}$, which means that the control $u$ steers the system from the initial state $\varphi$ to $x_{1}$ in time $a$, provided we can obtain a fixed point of the operator $\Psi$ which implies that the system is controllable. Here $\bar{x}:(-\infty, a] \rightarrow X$ is such that $\bar{x}_{0}=\varphi$ and $\bar{x}=x$ on $I$. From the axiom $(A)$ and our assumptions on $\varphi$, we infer that $\Psi x \in \mathcal{P C}$.

Next we claim that there exists $r>0$ such that $\Psi\left(B_{r}\left(y_{\left.\right|_{I}}, Y\right)\right) \subseteq B_{r}\left(y_{\left.\right|_{I}}, Y\right)$. If this property is false, then for every $r>0$, there exist $x^{r} \in B_{r}\left(y_{\left.\right|_{I}}, Y\right)$ and $t^{r} \in I$ such that $r<\left\|\Psi x^{r}\left(t^{r}\right)-y\left(t^{r}\right)\right\|$. By using Lemma 3.4, we get

$$
\begin{aligned}
r< & \left\|\Psi x^{r}\left(t^{r}\right)-y\left(t^{r}\right)\right\| \\
\leq & N H\|\varphi\|_{\mathcal{B}}+\tilde{N}\|\eta\|+\tilde{N} \int_{0}^{t^{r}} m(s) W\left(\| \overline{x^{r}}\right. \\
& +\tilde{\rho}\left(s,\left(\overline{x^{r}}\right)_{s}\right) \\
& +\tilde{N} M_{1} M_{2} \int_{0}^{t^{r}}\left[\left\|x_{1}\right\|+N H\|\varphi\|_{\mathcal{B}}+\tilde{N}\|\eta\|\right. \\
& +\tilde{N} \int_{0}^{a} m(s) W\left(\| \overline{x^{r}}\right. \\
&
\end{aligned}
$$




$$
\begin{aligned}
+ & \left.\left.\left\|I_{i}\left(y_{t_{i}}\right)\right\|\right)+\sum_{i=1}^{n} \tilde{N}\left(L_{J_{i}}\left\|\bar{x}_{t_{i}}-y_{t_{i}}\right\|_{\mathcal{B}}+\left\|J_{i}\left(y_{t_{i}}\right)\right\|\right)\right] d \xi \\
& +\sum_{i=1}^{n} N\left(L_{I_{i}}\left\|\bar{x}_{t_{i}}-y_{t_{i}}\right\|_{\mathcal{B}}+\left\|I_{i}\left(y_{t_{i}}\right)\right\|\right)+\sum_{i=1}^{n} \tilde{N}\left(L_{J_{i}}\left\|\bar{x}_{t_{i}}-y_{t_{i}}\right\|_{\mathcal{B}}+\left\|J_{i}\left(y_{t_{i}}\right)\right\|\right) \\
\leq & N H\|\varphi\|_{\mathcal{B}}+\tilde{N}\|\eta\|+\tilde{N} W\left(\left(M_{a}+J_{0}^{\varphi}\right)\|\varphi\|_{\mathcal{B}}+K_{a} r+K_{a}\|y\|_{a}\right) \int_{0}^{a} m(s) d s \\
& +a \tilde{N} M_{1} M_{2}\left[\left\|x_{1}\right\|+N H\|\varphi\|_{\mathcal{B}}+\tilde{N}\|\eta\|\right. \\
& +\tilde{N} W\left(\left(M_{a}+J_{0}^{\varphi}\right)\|\varphi\|_{\mathcal{B}}+K_{a} r+K_{a}\|y\|_{a}\right) \int_{0}^{a} m(s) d s \\
& \left.+\sum_{i=1}^{n} N\left(L_{I_{i}} K_{a} r+\left\|I_{i}\left(y_{t_{i}}\right)\right\|\right)+\sum_{i=1}^{n} \tilde{N}\left(L_{J_{i}} K_{a} r+\left\|J_{i}\left(y_{t_{i}}\right)\right\|\right)\right] \\
& +\sum_{i=1}^{n} N\left(L_{I_{i}} K_{a} r+\left\|I_{i}\left(y_{t_{i}}\right)\right\|\right)+\sum_{i=1}^{n} \tilde{N}\left(L_{J_{i}} K_{a} r+\left\|J_{i}\left(y_{t_{i}}\right)\right\|\right)
\end{aligned}
$$

and hence

$$
\left(1+a \tilde{N} M_{1} M_{2}\right)\left[K_{a}\left(\tilde{N} \Lambda \int_{0}^{a} m(s) d s+\sum_{i=1}^{n}\left(N L_{I_{i}}+\tilde{N} L_{J_{i}}\right)\right)\right] \geq 1
$$

which is contrary to our assumption.

Let $r>0$ be such that $\Psi\left(B_{r}\left(y_{\mid}, Y\right)\right) \subset B_{r}\left(y_{\left.\right|_{I}}, Y\right)$. In order to prove that $\Psi$ is a condensing map on $B_{r}\left(y_{\left.\right|_{I}}, Y\right)$ into $B_{r}\left(y_{\left.\right|_{I}}, Y\right)$. We introduce the decomposition $\Psi=\Psi_{1}+\Psi_{2}$ where

$$
\begin{aligned}
& \Psi_{1} x(t)=C(t) \varphi(0)+S(t) \eta+\sum_{0<t_{i}<t} C\left(t-t_{i}\right) I_{i}\left(\bar{x}_{t_{i}}\right)+\sum_{0<t_{i}<t} S\left(t-t_{i}\right) J_{i}\left(\bar{x}_{t_{i}}\right), \\
& \Psi_{2} x(t)=\int_{0}^{t} S(t-s)\left[f\left(s, \bar{x}_{\rho\left(s, \bar{x}_{s}\right)}\right)+B u(s)\right] d s .
\end{aligned}
$$

Now

$$
\begin{aligned}
\|B u(s)\| \leq & M_{1} M_{2}\left[\left\|x_{1}\right\|+N H\|\varphi\|_{\mathcal{B}}+\tilde{N}\|\eta\|+\tilde{N} \int_{0}^{a} \alpha_{r}(s) d s\right. \\
& \left.+N \sum_{i=1}^{n} \Phi_{i}\left\|\bar{x}_{t_{i}}\right\|+\tilde{N} \sum_{i=1}^{n} \Gamma_{i}\left\|\bar{x}_{t_{i}}\right\|\right] \\
\leq & M_{1} M_{2}\left[\left\|x_{1}\right\|+N H\left\|_{\varphi}\right\|_{\mathcal{B}}+\tilde{N}\|\eta\|+\tilde{N} \int_{0}^{a} \alpha_{r}(s) d s\right. \\
& \left.+\sum_{i=1}^{n} r\left(N \Phi_{i}+\tilde{N} \Gamma_{i}\right)\right]=A_{o} .
\end{aligned}
$$


Here we can apply the same technique that is used in Lemma 2.4. From the hypothesis $\left(\mathrm{H}_{\varphi}\right),(\mathrm{H} 1)$ and $(\mathrm{H} 2)$, we infer that $\Psi_{2}$ is completely continuous. Moreover, from the estimate

$$
\left\|\Psi_{1} x-\Psi_{1} z\right\|_{\mathcal{P C}} \leq K_{a} \sum_{i=1}^{n}\left(N L_{I_{i}}+\tilde{N} L_{J_{i}}\right)\|x-z\|_{\mathcal{P C}},
$$

it follows that $\Psi_{1}$ is a contraction on $B_{r}\left(y_{\left.\right|_{I}}, Y\right)$ which implies that $\Psi$ is a condensing operator on $B_{r}\left(y_{\left.\right|_{I}}, Y\right)$.

Finally, from Lemma $2.5, \Psi$ has a fixed point in $Y$. This means that any fixed point of $\Psi$ is a mild solution of the problem (1)-(4). This completes the proof.

\subsection{Second-order impulsive neutral systems}

In this section, we prove the result on controllability of nonlinear systems with state-dependent delay. Consider the impulsive neutral control system of the form

(9) $\frac{d}{d t}\left[x^{\prime}(t)-h\left(t, x_{t}\right)\right]=A x(t)+B u(t)+f\left(t, x_{\rho\left(t, x_{t}\right)}\right), t \in I=[0, a], t \neq t_{i}$,

$$
\begin{aligned}
x_{0} & =\varphi \in \mathcal{B}, \quad x^{\prime}(0)=\eta \in X, \\
\triangle x\left(t_{i}\right) & =I_{i}\left(x_{t_{i}}\right), i=1,2, \ldots, n, \\
\triangle x^{\prime}\left(t_{i}\right) & =J_{i}\left(x_{t_{i}}\right), i=1,2, \ldots, n,
\end{aligned}
$$

where $A, B, \rho, f, I_{i}$ and $J_{i}$ are defined as in equations (1)-(4). Here $h: I \times \mathcal{B} \rightarrow$ $X$ is an appropriate function. Furthermore we assume the following conditions:

(H5) The function $h: I \times \mathcal{B} \rightarrow X$ is completely continuous and there exists $L_{h}>0$ such that

$\left\|h\left(t, \psi_{1}\right)-h\left(t, \psi_{2}\right)\right\| \leq L_{h}\left\|\psi_{1}-\psi_{2}\right\|_{\mathcal{B}}, \quad\left(t, \psi_{i}\right) \in I \times \mathcal{B}, \quad i=1,2$.

(H6) There exist positive constants $c_{1}, c_{2}$ such that $\|h(t, \psi)\| \leq c_{1}\|\psi\|_{\mathcal{B}}+c_{2}$ for every $(t, \psi) \in I \times \mathcal{B}$.

Motivated by (7), let us start by defining what we mean by a mild solution of problem (9)-(12).

Definition 3.7. A function $x:(-\infty, a] \rightarrow X$ is called a mild solution of the impulsive abstract Cauchy problem (9)-(12), if $x_{0}=\varphi, x_{\rho\left(s, x_{s}\right)} \in \mathcal{B}$ for every $s \in I ;\left.x(\cdot)\right|_{I} \in \mathcal{P C}$ and

$$
\begin{aligned}
x(t)= & C(t) \varphi(0)+S(t)[\eta-h(0, \varphi)]+\int_{0}^{t} C(t-s) h\left(s, x_{s}\right) d s \\
& +\int_{0}^{t} S(t-s)\left[B u(s)+f\left(s, x_{\rho\left(s, x_{s}\right)}\right)\right] d s \\
& +\sum_{0<t_{i}<t} C\left(t-t_{i}\right) I_{i}\left(x_{t_{i}}\right)+\sum_{0<t_{i}<t} S\left(t-t_{i}\right) J_{i}\left(x_{t_{i}}\right), \quad t \in I .
\end{aligned}
$$


Theorem 3.8. Let the conditions $\left(\mathrm{H}_{\varphi}\right)$, (H1)-(H6) hold. Then the system (9)-(12) is controllable on $(-\infty, a]$ provided that

$$
\left(1+a \tilde{N} M_{1} M_{2}\right)\left[K_{a}\left(a N L_{h}+\tilde{N} \Lambda \int_{0}^{a} m(s) d s+\sum_{i=1}^{n}\left(N L_{I_{i}}+\tilde{N} L_{J_{i}}\right)\right)\right]<1 .
$$

Proof. Consider the space $Y=\{x \in \mathcal{P C}: u(0)=\varphi(0)\}$ endowed with the uniform convergence topology. Using the assumption (H2), for an arbitrary function $x(\cdot)$, we define the control

$$
\begin{aligned}
u(t)= & W^{-1}\left[x_{1}-C(a) \varphi(0)-S(a)[\eta-h(0, \varphi)]\right. \\
& -\int_{0}^{a} C(a-s) h\left(s, x_{s}\right) d s-\int_{0}^{a} S(a-s) f\left(s, x_{\rho\left(s, x_{s}\right)}\right) d s \\
& \left.-\sum_{i=1}^{n} C\left(a-t_{i}\right) I_{i}\left(x_{t_{i}}\right)-\sum_{i=1}^{n} S\left(a-t_{i}\right) J_{i}\left(x_{t_{i}}\right)\right](t) .
\end{aligned}
$$

Using this control, we shall show that the operator $\Psi: Y \rightarrow Y$ defined by

$$
\begin{aligned}
\Psi x(t)= & C(t) \varphi(0)+S(t)[\eta-h(0, \varphi)]+\int_{0}^{t} C(t-s) h\left(s, \bar{x}_{s}\right) d s \\
& +\int_{0}^{t} S(t-s) f\left(s, \bar{x}_{\rho\left(s, \bar{x}_{s}\right)}\right) d s+\int_{0}^{t} S(t-\xi) B W^{-1}\left[x_{1}\right. \\
& -C(a) \varphi(0)-S(a)[\eta-h(0, \varphi)]-\int_{0}^{a} C(a-s) h\left(s, \bar{x}_{s}\right) d s \\
& -\int_{0}^{a} S(a-s) f\left(s, \bar{x}_{\rho\left(s, \bar{x}_{s}\right)}\right) d s-\sum_{i=1}^{n} C\left(a-t_{i}\right) I_{i}\left(\bar{x}_{t_{i}}\right) \\
& \left.-\sum_{i=1}^{n} S\left(a-t_{i}\right) J_{i}\left(\bar{x}_{t_{i}}\right)\right](\xi) d \xi+\sum_{0<t_{i}<t} C\left(t-t_{i}\right) I_{i}\left(\bar{x}_{t_{i}}\right) \\
& +\sum_{0<t_{i}<t} S\left(t-t_{i}\right) J_{i}\left(\bar{x}_{t_{i}}\right), \quad t \in I,
\end{aligned}
$$

has a fixed point $x(\cdot)$. This fixed point $x(\cdot)$ is then a mild solution of the system (9)-(12). Clearly, $(\Psi x)(a)=x_{1}$, which means that the control $u$ steers the system from the initial state $\varphi$ to $x_{1}$ in time $a$, provided we can obtain a fixed point of the operator $\Psi$ which implies that the system is controllable. Here $\bar{x}:(-\infty, a] \rightarrow X$ is such that $\bar{x}_{0}=\varphi$ and $\bar{x}=x$ on $I$. From the axiom $(A)$ and our assumptions on $\varphi$, we infer that $\Psi x \in \mathcal{P C}$.

Next we prove that there exists $r>0$ such that $\Psi\left(B_{r}\left(y_{\left.\right|_{I}}, Y\right)\right) \subseteq B_{r}\left(y_{\left.\right|_{I}}, Y\right)$. If we assume that this property is false, then for every $r>0$, there exist $x^{r} \in B_{r}\left(y_{\left.\right|_{I}}, Y\right)$ and $t^{r} \in I$ such that $r<\left\|\Psi x^{r}\left(t^{r}\right)-y\left(t^{r}\right)\right\|$. Then, from 
Lemma 3.4, we find that

$$
\begin{aligned}
& r<\left\|\Psi x^{r}\left(t^{r}\right)-y\left(t^{r}\right)\right\| \\
& \leq N H\|\varphi\|_{\mathcal{B}}+\tilde{N}[\|\eta\|+\|h(0, \varphi)\|]+N \int_{0}^{t^{r}}\left\|h\left(s,\left(\overline{x^{r}}\right)_{s}\right)-h\left(s, y_{s}\right)\right\| d s \\
& +N \int_{0}^{t^{r}}\left\|h\left(s, y_{s}\right)\right\| d s+\tilde{N} \int_{0}^{t^{r}} m(s) W\left(\left\|\overline{x^{r}}{ }_{\rho\left(s,\left(\bar{x}^{r}\right)_{s}\right)}\right\|_{\mathcal{B}}\right) d s \\
& +\tilde{N} M_{1} M_{2} \int_{0}^{t^{r}}\left[\left\|x_{1}\right\|+N H\|\varphi\|_{\mathcal{B}}+\tilde{N}[\|\eta\|+\|h(0, \varphi)\|]\right. \\
& +N \int_{0}^{a}\left\|h\left(s, y_{s}\right)\right\| d s+N \int_{0}^{a}\left\|h\left(s,\left(\overline{x^{r}}\right)_{s}\right)-h\left(s, y_{s}\right)\right\| d s \\
& +\tilde{N} \int_{0}^{a} m(s) W\left(\left\|\overline{x^{r}} \rho\left(s,\left(\overline{x^{r}}\right)_{s}\right)\right\|_{\mathcal{B}}\right) d s+\sum_{i=1}^{n} N\left(L_{I_{i}}\left\|\bar{x}_{t_{i}}-y_{t_{i}}\right\|_{\mathcal{B}}+\left\|I_{i}\left(y_{t_{i}}\right)\right\|\right) \\
& \left.+\sum_{i=1}^{n} \tilde{N}\left(L_{J_{i}}\left\|\bar{x}_{t_{i}}-y_{t_{i}}\right\|_{\mathcal{B}}+\left\|J_{i}\left(y_{t_{i}}\right)\right\|\right)\right] d \xi \\
& +\sum_{i=1}^{n} N\left(L_{I_{i}}\left\|\bar{x}_{t_{i}}-y_{t_{i}}\right\|_{\mathcal{B}}+\left\|I_{i}\left(y_{t_{i}}\right)\right\|\right) \\
& +\sum_{i=1}^{n} \tilde{N}\left(L_{J_{i}}\left\|\bar{x}_{t_{i}}-y_{t_{i}}\right\|_{\mathcal{B}}+\left\|J_{i}\left(y_{t_{i}}\right)\right\|\right) \\
& \leq N H\|\varphi\|_{\mathcal{B}}+\tilde{N}[\|\eta\|+\|h(0, \varphi)\|]+N L_{h} K_{a} \int_{0}^{t^{r}}\left\|\overline{x^{r}}-y\right\|_{s} d s \\
& +N \int_{0}^{t^{r}}\left(c_{1}\left\|y_{s}\right\|_{\mathcal{B}}+c_{2}\right) d s+\tilde{N} W\left(\left(M_{a}+J_{0}^{\varphi}\right)\|\varphi\|_{\mathcal{B}}+K_{a} r+K_{a}\|y\|_{a}\right) \int_{0}^{a} m(s) d s \\
& +a \tilde{N} M_{1} M_{2}\left[\left\|x_{1}\right\|+N H\|\varphi\|_{\mathcal{B}}+\tilde{N}[\|\eta\|+\|h(0, \varphi)\|]+N L_{h} K_{a} \int_{0}^{a}\left\|\overline{x^{r}}-y\right\|_{s} d s\right. \\
& +N \int_{0}^{a}\left(c_{1}\left\|y_{s}\right\|_{\mathcal{B}}+c_{2}\right) d s+\tilde{N} W\left(\left(M_{a}+J_{0}^{\varphi}\right)\|\varphi\|_{\mathcal{B}}+K_{a} r+K_{a}\|y\|_{a}\right) \int_{0}^{a} m(s) d s \\
& \left.+\sum_{i=1}^{n} N\left(L_{I_{i}} K_{a} r+\left\|I_{i}\left(y_{t_{i}}\right)\right\|\right)+\sum_{i=1}^{n} \tilde{N}\left(L_{J_{i}} K_{a} r+\left\|J_{i}\left(y_{t_{i}}\right)\right\|\right)\right] \\
& +\sum_{i=1}^{n} N\left(L_{I_{i}} K_{a} r+\left\|I_{i}\left(y_{t_{i}}\right)\right\|\right)+\sum_{i=1}^{n} \tilde{N}\left(L_{J_{i}} K_{a} r+\left\|J_{i}\left(y_{t_{i}}\right)\right\|\right)
\end{aligned}
$$

and hence

$$
\left(1+a \tilde{N} M_{1} M_{2}\right)\left[K_{a}\left(a N L_{h}+\tilde{N} \Lambda \int_{0}^{a} m(s) d s+\sum_{i=1}^{n}\left(N L_{I_{i}}+\tilde{N} L_{J_{i}}\right)\right)\right] \geq 1
$$


which contradicts our assumption.

Let $r>0$ be such that $\Psi\left(B_{r}\left(y_{\mid I}, Y\right)\right) \subset B_{r}\left(y_{\mid}, Y\right)$. In order to prove that $\Psi$ is a condensing map on $B_{r}\left(y_{\left.\right|_{I}}, Y\right)$ into $B_{r}\left(y_{\mid}, Y\right)$. We introduce the decomposition $\Psi=\Psi_{1}+\Psi_{2}$ where

$$
\begin{aligned}
\Psi_{1} x(t)= & C(t) \varphi(0)+S(t)[\eta-h(0, \varphi)]+\int_{0}^{t} C(t-s) h\left(s, \bar{x}_{s}\right) d s \\
& +\sum_{0<t_{i}<t} C\left(t-t_{i}\right) I_{i}\left(\bar{x}_{t_{i}}\right)+\sum_{0<t_{i}<t} S\left(t-t_{i}\right) J_{i}\left(\bar{x}_{t_{i}}\right), \\
\Psi_{2} x(t)= & \int_{0}^{t} S(t-s)\left[f\left(s, \bar{x}_{\rho\left(s, \bar{x}_{s}\right)}\right)+B u(s)\right] d s .
\end{aligned}
$$

Now

$$
\begin{aligned}
\|B u(s)\| \leq & M_{1} M_{2}\left[\left\|x_{1}\right\|+N H\|\varphi\|_{\mathcal{B}}+\tilde{N}\left[\|\eta\|+c_{1}\|\varphi\|+c_{2}\right]\right. \\
& +N \int_{0}^{a}\left(c_{1}\left\|\bar{x}_{s}\right\|+c_{2}\right) d s+\tilde{N} \int_{0}^{a} \alpha_{r}(s) d s+N \sum_{i=1}^{n} \Phi_{i}\left\|\bar{x}_{t_{i}}\right\| \\
& \left.+\tilde{N} \sum_{i=1}^{n} \Gamma_{i}\left\|\bar{x}_{t_{i}}\right\|\right] \\
\leq & M_{1} M_{2}\left[\left\|x_{1}\right\|+N H\|\varphi\|_{\mathcal{B}}+\tilde{N}\left[\|\eta\|+c_{1}\|\varphi\|+c_{2}\right]\right. \\
& \left.+a N\left(c_{1} r+c_{2}\right)+\tilde{N} \int_{0}^{a} \alpha_{r}(s) d s+\sum_{i=1}^{n} r\left(N \Phi_{i}+\tilde{N} \Gamma_{i}\right)\right]=\tilde{A}_{o} .
\end{aligned}
$$

Here we can apply the same technique that is used in Lemma 2.4. From the hypothesis $\left(\mathrm{H}_{\varphi}\right),(\mathrm{H} 1)$ and $(\mathrm{H} 2)$, we infer that $\Psi_{2}$ is completely continuous. Moreover, from the estimate

$$
\begin{aligned}
\left\|\Psi_{1} x-\Psi_{1} z\right\|_{\mathcal{P C}} & \leq a N L_{h} K_{a}\|x-z\|_{\mathcal{P C}}+K_{a} \sum_{i=1}^{n}\left(N L_{I_{i}}+\tilde{N} L_{J_{i}}\right)\|x-z\|_{\mathcal{P C}} \\
& \leq K_{a}\left[a N L_{h}+\sum_{i=1}^{n}\left(N L_{I_{i}}+\tilde{N} L_{J_{i}}\right)\right]\|x-z\|_{\mathcal{P C}}
\end{aligned}
$$

it follows that $\Psi_{1}$ is a contraction on $B_{r}\left(y_{\mid}, Y\right)$ which implies that $\Psi$ is a condensing operator on $B_{r}\left(y_{\left.\right|_{I}}, Y\right)$.

Finally, from Sadovskii's fixed point theorem, $\Psi$ has a fixed point in $Y$. This means that any fixed point of $\Psi(\cdot)$ is a mild solution of the problem (9)-(12). This completes the proof. 
Theorem 3.9. Let the conditions $\left(\mathrm{H}_{\varphi}\right)$, (H1)-(H6) hold. Then the system (9)-(12) is controllable on $(-\infty, a]$ provided that

$$
\left(1+a \tilde{N} M_{1} M_{2}\right)\left[K_{a}\left(a N L_{h}+\tilde{N} \Lambda \int_{0}^{a} m(s) d s+\sum_{i=1}^{n}\left(N \zeta_{i}+\tilde{N} \sigma_{i}\right)\right)\right]<1 .
$$

Proof. Consider the space $Y=\{x \in \mathcal{P C}: u(0)=\varphi(0)\}$ endowed with the uniform convergence topology. Using the assumption (H2), for an arbitrary function $x(\cdot)$, we define the control

$$
\begin{aligned}
u(t)= & W^{-1}\left[x_{1}-C(a) \varphi(0)-S(a)[\eta-h(0, \varphi)]\right. \\
& -\int_{0}^{a} C(a-s) h\left(s, x_{s}\right) d s-\int_{0}^{a} S(a-s) f\left(s, x_{\rho\left(s, x_{s}\right)}\right) d s \\
& \left.-\sum_{i=1}^{n} C\left(a-t_{i}\right) I_{i}\left(x_{t_{i}}\right)-\sum_{i=1}^{n} S\left(a-t_{i}\right) J_{i}\left(x_{t_{i}}\right)\right](t) .
\end{aligned}
$$

Using this control, we shall show that the operator $\Psi: Y \rightarrow Y$ defined by

$$
\begin{aligned}
\Psi x(t)= & C(t) \varphi(0)+S(t)[\eta-h(0, \varphi)]+\int_{0}^{t} C(t-s) h\left(s, \bar{x}_{s}\right) d s \\
& +\int_{0}^{t} S(t-s) f\left(s, \bar{x}_{\rho\left(s, \bar{x}_{s}\right)}\right) d s+\int_{0}^{t} S(t-\xi) B W^{-1}\left[x_{1}\right. \\
& -C(a) \varphi(0)-S(a)[\eta-h(0, \varphi)]-\int_{0}^{a} C(a-s) h\left(s, \bar{x}_{s}\right) d s \\
& -\int_{0}^{a} S(a-s) f\left(s, \bar{x}_{\rho\left(s, \bar{x}_{s}\right)}\right) d s-\sum_{i=1}^{n} C\left(a-t_{i}\right) I_{i}\left(\bar{x}_{t_{i}}\right) \\
& \left.-\sum_{i=1}^{n} S\left(a-t_{i}\right) J_{i}\left(\bar{x}_{t_{i}}\right)\right](\xi) d \xi+\sum_{0<t_{i}<t} C\left(t-t_{i}\right) I_{i}\left(\bar{x}_{t_{i}}\right) \\
& +\sum_{0<t_{i}<t} S\left(t-t_{i}\right) J_{i}\left(\bar{x}_{t_{i}}\right), \quad t \in I,
\end{aligned}
$$

has a fixed point $x(\cdot)$. This fixed point $x(\cdot)$ is then a mild solution of the system (9)-(12). Clearly, $(\Psi x)(a)=x_{1}$, which means that the control $u$ steers the system from the initial state $\varphi$ to $x_{1}$ in time $a$, provided we can obtain a fixed point of the operator $\Psi$ which implies that the system is controllable. Here $\bar{x}:(-\infty, a] \rightarrow X$ is such that $\bar{x}_{0}=\varphi$ and $\bar{x}=x$ on $I$. From the axiom $(A)$ and our assumptions on $\varphi$, we infer that $\Psi x \in \mathcal{P C}$.

Next we prove that there exists $r>0$ such that $\Psi\left(B_{r}\left(y_{\left.\right|_{I}}, Y\right)\right) \subseteq B_{r}\left(y_{\left.\right|_{I}}, Y\right)$. If we assume that this property is false, then for every $r>0$, there exist $x^{r} \in B_{r}\left(y_{\left.\right|_{I}}, Y\right)$ and $t^{r} \in I$ such that $r<\left\|\Psi x^{r}\left(t^{r}\right)-y\left(t^{r}\right)\right\|$. Then, from 
Lemma 3.4, we get

$$
\begin{aligned}
& r<\left\|\Psi x^{r}\left(t^{r}\right)-y\left(t^{r}\right)\right\| \\
& \leq N H\|\varphi\|_{\mathcal{B}}+\tilde{N}[\|\eta\|+\|h(0, \varphi)\|]+N \int_{0}^{t^{r}}\left\|h\left(s,\left(\overline{x^{r}}\right)_{s}\right)-h\left(s, y_{s}\right)\right\| d s \\
& +N \int_{0}^{t^{r}}\left\|h\left(s, y_{s}\right)\right\| d s+\tilde{N} \int_{0}^{t^{r}} m(s) W\left(\left\|\overline{x^{r}} \rho\left(s,\left(\bar{x}^{r}\right)_{s}\right)\right\|_{\mathcal{B}}\right) d s \\
& +\tilde{N} M_{1} M_{2} \int_{0}^{t^{r}}\left[\left\|x_{1}\right\|+N H\|\varphi\|_{\mathcal{B}}+\tilde{N}[\|\eta\|+\|h(0, \varphi)\|]+N \int_{0}^{a}\left\|h\left(s, y_{s}\right)\right\| d s\right. \\
& +N \int_{0}^{a}\left\|h\left(s,\left(\overline{x^{r}}\right)_{s}\right)-h\left(s, y_{s}\right)\right\| d s+\tilde{N} \int_{0}^{a} m(s) W\left(\left\|\overline{x^{r}} \rho\left(s,\left(\overline{x^{r}}\right)_{s}\right)\right\|_{\mathcal{B}}\right) d s \\
& \left.+N \sum_{i=1}^{n}\left\|I_{i}\left(\bar{x}_{t_{i}}\right)\right\|+\tilde{N} \sum_{i=1}^{n}\left\|J_{i}\left(\bar{x}_{t_{i}}\right)\right\|\right] d \xi+N \sum_{i=1}^{n}\left\|I_{i}\left(\bar{x}_{t_{i}}\right)\right\|+\tilde{N} \sum_{i=1}^{n}\left\|J_{i}\left(\bar{x}_{t_{i}}\right)\right\| \\
& \leq N H\|\varphi\|_{\mathcal{B}}+\tilde{N}[\|\eta\|+\|h(0, \varphi)\|]+N L_{h} K_{a} \int_{0}^{t^{r}}\left\|\overline{x^{r}}-y\right\|_{s} d s \\
& +N \int_{0}^{t^{r}}\left(c_{1}\left\|y_{s}\right\|_{\mathcal{B}}+c_{2}\right) d s+\tilde{N} W\left(\left(M_{a}+J_{0}^{\varphi}\right)\|\varphi\|_{\mathcal{B}}+K_{a} r+K_{a}\|y\|_{a}\right) \int_{0}^{a} m(s) d s \\
& +a \tilde{N} M_{1} M_{2}\left[\left\|x_{1}\right\|+N H\|\varphi\|_{\mathcal{B}}+\tilde{N}[\|\eta\|+\|h(0, \varphi)\|]+N L_{h} K_{a} \int_{0}^{a}\left\|\overline{x^{r}}-y\right\|_{s} d s\right. \\
& +N \int_{0}^{a}\left(c_{1}\left\|y_{s}\right\|_{\mathcal{B}}+c_{2}\right) d s+\tilde{N} W\left(\left(M_{a}+J_{0}^{\varphi}\right)\|\varphi\|_{\mathcal{B}}+K_{a} r+K_{a}\|y\|_{a}\right) \int_{0}^{a} m(s) d s \\
& \left.+N \sum_{i=1}^{n} \Phi_{i}\left(\left\|\bar{x}_{t_{i}}\right\|_{\mathcal{B}}\right)+\tilde{N} \sum_{i=1}^{n} \Gamma_{i}\left(\left\|\bar{x}_{t_{i}}\right\|_{\mathcal{B}}\right)\right] \\
& +N \sum_{i=1}^{n} \Phi_{i}\left(\left\|\bar{x}_{t_{i}}\right\|_{\mathcal{B}}\right)+\tilde{N} \sum_{i=1}^{n} \Gamma_{i}\left(\left\|\bar{x}_{t_{i}}\right\|_{\mathcal{B}}\right) .
\end{aligned}
$$

Since $\Phi_{i}$ and $\Gamma_{i}$ are nondecreasing operators, we have

$$
\begin{aligned}
r \leq & N H\|\varphi\|_{\mathcal{B}}+\tilde{N}[\|\eta\|+\|h(0, \varphi)\|]+N L_{h} K_{a} \int_{0}^{t^{r}}\left\|\overline{x^{r}}-y\right\|_{s} d s \\
& +N \int_{0}^{t^{r}}\left(c_{1}\left\|y_{s}\right\|_{\mathcal{B}}+c_{2}\right) d s+\tilde{N} W\left(\left(M_{a}+J_{0}^{\varphi}\right)\|\varphi\|_{\mathcal{B}}+K_{a} r+K_{a}\|y\|_{a}\right) \int_{0}^{a} m(s) d s \\
& +a \tilde{N} M_{1} M_{2}\left[\left\|x_{1}\right\|+N H\|\varphi\|_{\mathcal{B}}+\tilde{N}[\|\eta\|+\|h(0, \varphi)\|]+N L_{h} K_{a} \int_{0}^{a}\left\|\overline{x^{r}}-y\right\|_{s} d s\right. \\
& +N \int_{0}^{a}\left(c_{1}\left\|y_{s}\right\|_{\mathcal{B}}+c_{2}\right) d s+\tilde{N} W\left(\left(M_{a}+J_{0}^{\varphi}\right)\|\varphi\|_{\mathcal{B}}+K_{a} r+K_{a}\|y\|_{a}\right) \int_{0}^{a} m(s) d s
\end{aligned}
$$




$$
\left.+N \sum_{i=1}^{n} \Phi_{i}\left(r^{*}\right)+\tilde{N} \sum_{i=1}^{n} \Gamma_{i}\left(r^{*}\right)\right]+N \sum_{i=1}^{n} \Phi_{i}\left(r^{*}\right)+\tilde{N} \sum_{i=1}^{n} \Gamma_{i}\left(r^{*}\right),
$$

where $\left\|\bar{x}_{t_{i}}\right\|_{\mathcal{B}} \leq r^{*}=\left(M_{a}+J_{0}^{\varphi}\right)\|\varphi\|_{\mathcal{B}}+K_{a}\left(r+\|y\|_{a}\right)$ and hence

$$
\left(1+a \tilde{N} M_{1} M_{2}\right)\left[K_{a}\left(a N L_{h}+\tilde{N} \Lambda \int_{0}^{a} m(s) d s+\sum_{i=1}^{n}\left(N \zeta_{i}+\tilde{N} \sigma_{i}\right)\right)\right] \geq 1,
$$

which contradicts our assumption.

Arguing as in the proof of Theorem 3.8, we can prove that $\Psi(\cdot)$ is a condensing map on $B_{r}\left(y_{\left.\right|_{I}}, Y\right)$ and, from Lemma 2.5, we conclude that there exists a mild solution $x(\cdot)$ for $(9)-(12)$. The proof is complete.

Remark 3.10. Similar results to those of sub-linear growth cases [6, Corollary $3.1,3.2$ and 3.3] hold for controllability results.

\section{Examples}

In this section, we consider some applications for our results. We choose the space $X=L^{2}([0, \pi]), \mathcal{B}=\mathcal{P C}_{0} \times L^{2}(h, X)$ is the space introduced in Example 2.2 and $A: D(A) \subset X \rightarrow X$ is the operator defined by $A u=u^{\prime \prime}$ with domain $D(A)=\left\{u \in X, u^{\prime \prime} \in X, u(0)=u(\pi)=0\right\}$. It is well known that $A$ is the infinitesimal generator of a strongly continuous cosine function $(C(t))_{t \in \mathbb{R}}$ on $X$. Moreover $A$ has a discrete spectrum with eigenvalues of the form $-n^{2}$, $n \in \mathbb{N}$, and the corresponding normalized eigenfunctions given by $z_{n}(\zeta):=$ $\left(\frac{2}{\pi}\right)^{\left(\frac{1}{2}\right)} \sin (n \zeta)$. Also the following properties hold:

(a) The set of functions $\left\{z_{n}: n \in \mathbb{N}\right\}$ forms an orthonormal basis of $X$.

(b) If $x \in D(A)$, then $A x=-\sum_{n=1}^{\infty} n^{2}\left\langle x, z_{n}\right\rangle z_{n}$.

(c) For $x \in X, C(t) x=\sum_{n=1}^{\infty} \cos (n t)\left\langle x, z_{n}\right\rangle z_{n}$ and the associated sine family is $S(t) x=\sum_{n=1}^{\infty} \frac{\sin (n t)}{n}\left\langle x, z_{n}\right\rangle z_{n}$.

(d) If $G$ is the group of translations on $X$ defined by $G(t) x(\zeta)=\tilde{x}(\zeta+$ $t$ ), where $\tilde{x}(\cdot)$ is the extension of $x(\cdot)$ with period $2 \pi$, then $C(t)=$ $\frac{1}{2}[G(t)+G(-t)]$. Hence it follows, see [10], that $A=B^{2}$ where $B$ is the infinitesimal generator of $G$ and $E=\left\{x \in H^{1}(0, \pi): x(0)=x(\pi)=0\right\}$.

\subsection{Second order system}

Consider the following impulsive differential equation with state-dependent delay and control

$$
\begin{aligned}
\frac{\partial^{2}}{\partial t^{2}} w(t, \xi)= & \frac{\partial^{2}}{\partial \xi^{2}} w(t, \xi)+\mu(t, \xi) \\
& +\int_{-\infty}^{t} a(s-t) w\left(s-\rho_{1}(t) \rho_{2}(\|w(t)\|), \xi\right) d s
\end{aligned}
$$

for $t \in I=[0, a], \xi \in[0, \pi]$, subject to the initial conditions

$$
w(t, 0)=w(t, \pi)=0, \quad t \in I=[0, a],
$$




$$
\begin{aligned}
w(\tau, \xi) & =\varphi(\tau, \xi), \quad \tau \leq 0, \quad 0 \leq \xi \leq \pi \\
\triangle w\left(t_{i}\right)(\xi) & =\int_{-\infty}^{t_{i}} b_{i}\left(t_{i}-s\right) w(s, \xi) d s, \quad i=1,2, \ldots, n, \\
\triangle w^{\prime}\left(t_{i}\right)(\xi) & =\int_{-\infty}^{t_{i}} \tilde{b}_{i}\left(t_{i}-s\right) w(s, \xi) d s, \quad i=1,2, \ldots, n .
\end{aligned}
$$

We have to show that there exists a control $\mu$ which steers (13) from any specified initial state to the final state in a Banach space $X$.

To do this, let $\varphi \in \mathcal{B}$, the functions $\rho_{i}:[0, \infty) \rightarrow[0, \infty), i=1,2 ; a: \mathbb{R} \rightarrow \mathbb{R}$ be continuous, $L_{f}=\left(\int_{-\infty}^{0} \frac{\left(a^{2}(s)\right)}{g(s)} d s\right)^{\frac{1}{2}}<\infty$ and the following condition hold:

(a) The functions $b_{i}, \tilde{b}_{i} \in C(\mathbb{R}, \mathbb{R})$ and $L_{I_{i}}:=\left(\int_{-\infty}^{0} \frac{b_{i}^{2}(s)}{g(s)} d s\right)^{\frac{1}{2}}, L_{J_{i}}=$ $\left(\int_{-\infty}^{0} \frac{\tilde{b}_{i}^{2}(s)}{g(s)} d s\right)^{\frac{1}{2}}, i=1, \ldots, n$, are finite.

Assume that the bounded linear operator $B: U \subset I \rightarrow X$ is defined by

$$
(B u)(t)(\xi)=\mu(t, \xi), \quad \xi \in[0, \pi] .
$$

Define the operators $f: I \times \mathcal{B} \rightarrow X, \rho: I \times \mathcal{B} \rightarrow X$ and $I_{i}, J_{i}: \mathcal{B} \rightarrow X$ by

$$
\begin{aligned}
f(t, \psi)(\xi) & =\int_{-\infty}^{0} a(s) \psi(s, \xi) d s, \\
\rho(s, \psi) & =s-\rho_{1}(s) \rho_{2}(\|\psi(0)\|), \\
I_{i}(\psi)(\xi) & =\int_{-\infty}^{0} b_{i}(-s) \psi(s, \xi) d s, \quad i=1,2, \ldots, n, \\
J_{i}(\psi)(\xi) & =\int_{-\infty}^{0} \tilde{b}_{i}(-s) \psi(s, \xi) d s, \quad i=1,2, \ldots, n .
\end{aligned}
$$

With the choice of $A, B, f, \rho, I_{i}$ and $J_{i},(1)$-(4) is the abstract formulation of (13). Now the linear operator $W$ is given by

$$
(W u)(\xi)=\sum_{n=1}^{\infty} \int_{0}^{\pi} \frac{1}{n} \sin n s\left(\mu(s, \xi), z_{n}\right) z_{n} d s, \quad \xi \in[0, \pi] .
$$

Assume that this operator has a bounded inverse $W^{-1}$ in $L^{2}(I, U) / \operatorname{Ker} W$. Moreover the functions $f, I_{i}$ and $J_{i}, i=1,2, \ldots, n$ are bounded linear operators with $\|f(t, \cdot)\|_{\mathcal{L}(\mathcal{B}, X)} \leq L_{f},\left\|I_{i}\right\|_{\mathcal{L}(\mathcal{B}, X)} \leq L_{I_{i}},\left\|J_{i}\right\|_{\mathcal{L}(\mathcal{B}, X)} \leq L_{J_{i}}$. Hence the second order impulsive system (13) is controllable.

\subsection{Second order neutral system}

Consider the following impulsive neutral differential equation with statedependent delay and control

$$
\frac{\partial}{\partial t}\left[\frac{\partial}{\partial t} w(t, \xi)+\int_{-\infty}^{t} \int_{0}^{\pi} b(t-s, \eta, \xi) w(s, \eta) d \eta d s\right]=\frac{\partial^{2}}{\partial \xi^{2}} w(t, \xi)
$$




$$
+\mu(t, \xi)+\int_{-\infty}^{t} a(s-t) w\left(s-\rho_{1}(t) \rho_{2}(\|w(t)\|), \xi\right) d s,
$$

for $t \in I=[0, a], \xi \in[0, \pi]$, subject to the initial conditions

$$
\begin{aligned}
w(t, 0) & =w(t, \pi)=0, \quad t \in I, \\
\frac{\partial}{\partial t} w(0, \xi) & =\xi(\pi), \\
w(\tau, \xi) & =\varphi(\tau, \xi), \quad \tau \leq 0, \quad 0 \leq \xi \leq \pi, \\
\triangle w\left(t_{i}\right)(\xi) & =\int_{-\infty}^{t_{i}} b_{i}\left(t_{i}-s\right) w(s, \xi) d s, \quad i=1,2, \ldots, n, \\
\triangle w^{\prime}\left(t_{i}\right)(\xi) & =\int_{-\infty}^{t_{i}} \tilde{b}_{i}\left(t_{i}-s\right) w(s, \xi) d s, \quad i=1,2, \ldots, n,
\end{aligned}
$$

where $\varphi, B, a, \rho_{i}, i=1,2$ and $L_{f}$ are as defined in Example 4.1. Assume that the condition (a) of the previous example holds and that

(b) The functions $b(s, \eta, \xi), \frac{\partial b(s, \eta, \xi)}{\partial \xi}$ are continuous and measurable, $b(s, \eta, \pi)$ $=b(s, \eta, 0)=0$ and

$$
L_{h}=\max \left\{\left(\int_{0}^{\pi} \int_{-\infty}^{0} \int_{0}^{\pi} \frac{1}{g(s)}\left(\frac{\partial^{i} b(s, \eta, \xi)}{\partial \xi^{i}}\right) d \eta d s d \xi\right)^{\frac{1}{2}}: i=0,1\right\}<\infty .
$$

Define the functions $A, B, f, \rho, I_{i}, J_{i}$ and $W$ as in Example 4.1 and the map $h: I \times \mathcal{B} \rightarrow X$ by

$$
h(\psi)(\xi)=\int_{-\infty}^{0} \int_{0}^{\pi} b(s, \nu, \xi) \psi(s, \nu) d \nu d s .
$$

With the choice of $A, B, W, h, f, \rho, I_{i}$ and $J_{i}$, the system (14) can be modelled as (9)-(12). Moreover the function $h$ is a bounded linear operator with $\|h(t, \cdot)\|_{\mathcal{L}(\mathcal{B}, X)} \leq L_{h}$. Hence the second order impulsive neutral system (14) is controllable.

Acknowledgement. The first author is thankful to UGC, New Delhi for providing BSR-Fellowship during 2010.

\section{References}

[1] W. G. Aiello, H. I. Freedman, and J. Wu, Analysis of a model representing stagestructured population growth with state-dependent time delay, SIAM J. Appl. Math. 52 (1992), no. 3, 855-869.

[2] A. Anguraj, M. M. Arjunan, and E. Hernandez, Existence results for an impulsive neutral functional differential equation with state-dependent delay, Appl. Anal. 86 (2007), no. $7,861-872$.

[3] K. Balachandran and S. M. Anthoni, Controllability of second-order semilinear neutral functional differential systems in Banach spaces, Comput. Math. Appl. 41 (2001), no. 10-11, 1223-1235.

[4] M. Bartha, Periodic solutions for differential equations with state-dependent delay and positive feedback, Nonlinear Anal. 53 (2003), no. 6, 839-857. 
[5] Y. Cao, J. Fan, and T. C. Gard, The effects of state-dependent time delay on a stagestructured population growth model, Nonlinear Anal. 19 (1992), no. 2, 95-105.

[6] Y.-K. Chang, M. M. Arjunan, and V. Kavitha, Existence results for a second order impulsive functional differential equation with state-dependent delay, Differ. Equ. Appl. 1 (2009), no. 3, 325-339.

[7] F. Chen, D. Sun, and J. Shi, Periodicity in a food-limited population model with toxicants and state-dependent delays, J. Math. Anal. Appl. 288 (2003), no. 1, 136-146.

[8] A. Domoshnitsky, M. Drakhlin, and E. Litsyn, On equations with delay depending on solution, Nonlinear Anal. 49 (2002), no. 5, 689-701.

[9] R. D. Driver, A neutral system with state-dependent delay, J. Differential Equations 54 (1984), no. 1, 73-86.

[10] H. O. Fattorini, Second Order Linear Differential Equations in Banach Spaces, NorthHolland, Amsterdam, 1985.

[11] F. Hartung, Parameter estimation by quasilinearization in functional differential equations with state-dependent delays: a numerical study, Proceedings of the Third World Congress of Nonlinear Analysts, Part 7 (Catania, 2000), Nonlinear Anal. 47 (2001), no. 7, 4557-4566.

[12] F. Hartung, T. L. Herdman, and J. Turi, Parameter identification in classes of neutral differential equations with state-dependent delays, Nonlinear Anal. 39 (2000), no. 3, 305-325.

[13] F. Hartung, T. Krisztin, H.-O. Walther, and J. Wu, Functional Differential Equations with State-Dependent Delays: Theory and Applications, in: A. Canada, P. Drabek, A. Fonda (Eds.), Handbook of Differential Equations : Ordinary Differential Equations, Elsevier B. V, 2006

[14] F. Hartung and J. Turi, Identification of parameters in delay equations with statedependent delays, Nonlinear Anal. 29 (1997), no. 11, 1303-1318.

[15] E. Hernandez, Existence of solutions for a second order abstract functional differential equation with state-dependent delay, Electron. J. Differential Equations 2007 (2007), no. 21,10 pp.

[16] E. Hernandez and M. McKibben, On state-dependent delay partial neutral functional differential equations, Appl. Math. Comput. 186 (2007), no. 1, 294-301.

[17] E. Hernandez, M. A. McKibben, and H. Henriquez, Existence results for partial neutral functional differential equations with state-dependent delay, Math. Comput. Modelling 49 (2009), no. 5-6, 1260-1267.

[18] E. Hernandez, M. Pierri, and G. Uniao, Existence results for an impulsive abstract partial differential equation with state-dependent delay, Comput. Math. Appl. 52 (2006), no. 3-4, 411-420.

[19] E. Hernandez, A. Prokopczyk, and L. A. Ladeira, A note on partial functional differential equations with state-dependent delay, Nonlinear Anal. Real World Appl. 7 (2006), no. 4, 510-519.

[20] E. Hernandez, R. Sakthivel, and S. Tanaka, Existence results for impulsive evolution differential equations with state-dependent delay, Electron. J. Differential Equations 2008 (2008), no. 28, 11 pp.

[21] Y. Hino, S. Murakami, and T. Naito, Functional Differential Equations with Infinite Delay, Lecture Notes in Mathematics, 1473, Springer-Verlag, Berlin, 1991.

[22] J. M. Jeong and H. G. Kim, Controllability for semilinear functional integrodifferential equations, Bull. Korean Math. Soc. 46 (2009), no. 3, 463-475.

[23] J. Kisynski, On cosine operator functions and one parameter group of operators, Studia Math. 49 (1972), 93-105.

[24] V. Lakshmikantham, D. D. Bainov, and P. S. Simeonov, Theory of Impulsive Differential Equations, World Scientific, Singapore, 1989. 
[25] W. S. Li, Y.-K. Chang, and J. J. Nieto, Solvability of impulsive neutral evolution differential inclusions with state-dependent delay, Math. Comput. Modelling 49 (2009), no. 9-10, 1920-1927.

[26] S. K. Ntouyas and D. O' Regan, Some remarks on controllability of evolution equations in Banach spaces, Electron. J. Differential Equations 2009 (2009), no. 79, 6 pp.

[27] J. Y. Park and H. K. Han, Controllability for some second order differential equations, Bull. Korean Math. Soc. 34 (1997), no. 3, 411-419.

[28] J. Y. Park, S. H. Park, and Y. H. Kang, Controllability of second-order impulsive neutral functional differential inclusions in Banach spaces, Math. Methods Appl. Sci. 33 (2010), no. 3, 249-262.

[29] B. N. Sadovskii, On a fixed point principle, Funct. Anal. Appl. 1 (1967), no. 2, 74-76.

[30] R. Sakthivel, N. I. Mahmudov, and J. H. Kim, On controllability of second-order nonlinear impulsive differential systems, Nonlinear Anal. 71 (2009), no. 1-2, 45-52.

[31] A. M. Samoilenko and N. A. Perestyuk, Impulsive Differential Equations, World Scientific, Singapore, 1995.

[32] J. G. Si and X. P. Wang, Analytic solutions of a second-order functional differential equation with a state dependent delay, Results Math. 39 (2001), no. 3-4, 345-352.

[33] C. C. Travis and G. F. Webb, Compactness, regularity and uniform continuity properties of strongly continuous cosine families, Houston J. Math. 3 (1977), no. 4, 555-567.

[34] Cosine families and abstract nonlinear second order differential equations, Acta Math. Acad. Sci. Hungar. 32 (1978), no. 1-2, 75-96.

[35] Z. Yang and J. Cao, Existence of periodic solutions in neutral state-dependent delay equations and models, J. Comput. Appl. Math. 174 (2005), no. 1, 179-199.

Ganesan ARthi

Department of Mathematics

BHARATHIAR UNIVERSITY

Coimbatore 641 046, INDiA

E-mail address: arthimath@gmail.com

Krishnan BaLachandran

Department of Mathematics

BHARATHIAR UNIVERSITY

CoImbatore 641 046, India

E-mail address: balachandran-k@lycos.com 